\title{
The reach, adoption, and effectiveness of online training for healthcare professionals.
}

\section{Short communication}

Traditionally the continued professional development of healthcare professionals is completed through classroom based educational courses, workshops, and conferences. These can prove costly and time intensive. Online learning is becoming increasingly common, is easy to access, and can save learners time which is important in a healthcare system where job demands are high and study leave is limited. Well-designed internetbased learning has been shown to be as effective as traditional classroom based learning in the skill and knowledge development of healthcare professionals, ${ }^{1}$ however the reach, adoption, and effectiveness of such training is largely underreported in the literature.

This short communication reports on evaluation data from delivery of a 60 minute online training session to healthcare professionals, between January and November 2016.

\section{The online training}

The online training was developed by Webb, Foster and Poulter $(2016)^{2}$ to overcome an identified knowledge gap in nurses regarding the importance of physical activity for cancer patients. ${ }^{3}$ Healthcare professionals are expected to give lifestyle advice ${ }^{4}$ yet in a UK survey of 15,524 colorectal cancer patients, only $31 \%$ recall receiving such advice. ${ }^{5}$

Developed using behaviour change theory, ${ }^{6}$ the training, with associated follow-up, aims to improve the capability, opportunity and motivation of nurses to give very brief advice on physical activity to their cancer patients. Details of training development and feasibility have been reported previously. ${ }^{2,7}$ The original training was designed specifically for nurses however, a recommendation from the feasibility study was that it be open to all healthcare professionals engaging with cancer patients. ${ }^{2}$

Online seminar technology is used to deliver the training, providing a virtual medium for peer-to-peer interaction, important for learner engagement and social learning. ${ }^{8}$ All healthcare professionals attending the online training are asked to complete a baseline survey on their discussions of physical activity with cancer patients, rating discussions as 0 $25 \%$ of the time, $26-50 \%, 51-75 \%$ or $76-100 \%$ of the time. A similar survey is administered again 12 weeks later to monitor changes in the frequency of discussions on physical activity. 
The capability, opportunity, and motivation of learners to raise physical activity with their cancer patients is measured using a 10-item survey administered at the end of the training. This uses a 5 point Likert scale, with 5 representing a strong agreement to improvements because of the training, and 1 representing a strong disagreement.

\section{The RE-AIM framework}

The RE-AIM framework ${ }^{9}$ has been used to analyse the evaluation data in the following way:-

- The REACH of the training is reported in relation to the contacts made from promotional activity and subsequent bookings, and training completions.

- The EFFECTIVENESS of the training is suggested from self-reported changes in the frequency of discussions of physical activity with cancer patients before, and 12 weeks after training completion. These results are reported in relation to the capability, opportunity, and motivation of learners to give physical activity advice.

- The ADOPTION of the training relates to the bookings received from specific healthcare professions.

- The IMPLEMETATION and MAINTENACE domains of the RE-AIM framework are not covered within this communication.

The analysis of these areas is presented in figure 1.

\section{Reach}

The training was promoted throughout the 11 month period from January to November 2016 . It was promoted electronically to a network of 6,344 healthcare professionals held by Macmillan Cancer Support, via their 'Mac Mail' e-newsletter. During the promotional period, this e-newsletter had an open rate of 1,802 healthcare professionals. Across two separate enewsletter articles, 67 and 111 healthcare professionals clicked on a link associated with the training, a $3.7 \%$ and $6.2 \%$ response rate respectively for those that opened the e-newsletter, and $1.1 \%$ and $1.7 \%$ total response rate respectively.

The online training was promoted on a 'consequences of cancer treatment' webpage on doctors.net.uk, the UKs largest professional network for doctors. Over the promotional period 4,742 unique visits were made to this page, which resulted in 23 accessing information regarding the training, a $0.5 \%$ response rate. The training was promoted in two articles on a 'Nursing Times' microsite, ${ }^{10}$ which has a monthly reach of 538 unique visitors. It is not known how many of these visitors accessed these articles. Finally, the online training 
was promoted at the 2016 National Cancer Research Institute conference, with promotional materials being placed within 600 delegate packs.

The total reach of this promotional activity combined is estimated to be 7,682 healthcare professionals. Two-hundred and seven healthcare professionals registered to attend one of 11 possible training sessions (one available per month), a ratio of 38 contacts to 1 booking.

\section{Adoption}

The largest number of bookings came from Nurses with 72 registrations (35\%), the professional group for whom the training was originally developed. This was followed by Support Workers $(n=31,15 \%)$, Physiotherapists $(n=29,14 \%)$, Radiation Therapists $(n=27$; $13 \%)$, Dietitians ( $n=15,7.2 \%)$, and Occupational therapists $(n=10,4.8 \%)$, with small numbers from other professions $(n=23)$. It is noted that most bookings came from groups that would be categorised as Allied Healthcare Professionals.

Of this 207,127 completed the hour-long training, a completion rate of $61.4 \%$. The highest booking to completion figures were observed in Dietitians with a completion rate of $73.3 \%$ ( $n=11$ of 15$)$, followed by Physiotherapists ( $n=20$ of $29,69 \%)$, Support Workers $(n=21$ of $31,67.7 \%$ ), Nurses ( $n=44$ of $72,61.1 \%)$, Occupational Therapists ( $n=5$ of $10,50 \%)$, and Radiation Therapist ( $n=13$ of $27,48.2 \%$ ).

\section{Effectiveness}

Of the 127 healthcare professionals who completed the training, 45 declined to complete the baseline survey on their discussions of physical activity with cancer patients. Fifty-three $(64.6 \%)$ of the 82 learners who provided baseline data were discussing physical activity with their cancer patients less than $75 \%$ of the time.

Only 44 of the 82 learners who provided baseline data responded to the 12 week follow-up survey, despite learners receiving four reminders. Of those that responded, 19 maintained discussions at the highest level (76 to $100 \%$ of the time), 16 improved their discussions from baseline, six maintained discussions at a lower frequency, and three reported discussing physical activity less. Intention to treat analysis, using the Wilcoxon signed ranks test, two tailed, to an alpha of 0.05 with missing data at 12 weeks follow up assumed to show no change, reports a significant increase in the frequency of discussion on physical activity $(p \leq 0.01)$. 
Thirty one of the 44 who provided data 12 weeks after the training, also completed the post training survey on their capability, opportunity, and motivation to give physical activity advice. The capability, opportunity, and motivation scores of those that either maintained discussions at the highest level, or improved from baseline, were 4.32/5, 4.35/5, and 4.43/5 respectively ( $n=25$ of 31 ). Those that maintained a lower frequency of discussion, or reduced their discussion from baseline, reported capability, opportunity, and motivation scores of $4.08 / 5,4.17 / 5$, and $4 / 5$ respectively ( $n=6$ of 31 ), a difference of $0.24,0.18$, and 0.43 . As the sample is small, it is not possible to draw firm conclusions, but the differences in these capability, opportunity, and motivation scores warrants further investigation to help identify the predictors of behaviour change, or maintenance of the desired behaviour in training attendees.

\section{Summary}

Whilst any conclusions should be approached with caution due to the limitations in data collection at each data point, the data that is available offers some insight into areas that warrant further investigation. These are, the importance of motivation over capability and opportunity of learners in predicting a change in practice, or maintenance of best practice; the popularity of such online training with certain healthcare professions and not others; the large number of healthcare professional contacts required to result in a training booking, and how to improve booking to completion rates. Online training offers promised, but these areas need to be understood to ensure that it is developed, promoted, and delivered effectively to change practice. The RE-AIM framework is a useful tool to support the evaluation of online training.

This service evaluation did not receive any specific grant from funding agencies in the public, commercial, or not-for-profit sectors. Delivery of the online training was funded by Macmillan Cancer Support. As this short communication reports on service evaluation data, ethical approval was not sought.

\section{Key words}

Online training; Very brief advice; Physical activity.

\section{References}

1. Hemmati N, Omrani S, Hemmati N. A comparison of internet-based learning and traditional classroom lecture to learn CPR for continuing medical education. Turkish Online Journal of Distance Education 2013; 14 (1):256-265.

2. Webb J, Hall J, Hall K, Fabunmi-Alade R. Increasing the frequency of physical 
activity very brief advice by nurses to cancer patients. A mixed methods feasibility study of a training intervention. Public Health. Elsevier 2016; 139: 121-131.

3. Anderson AS, Caswell S, Wells M, Steele RJC. Obesity and lifestyle advice in colorectal cancer survivors - how well are clinicians prepared? Colorectal Dis [Internet]. 2013;15(8):949-57. Available from:

http://www.scopus.com/inward/record.url?eid=2-s2.0-

84883221738\&partnerID=40\&md5=eaffc9fcba1632abefb4315bc976f0f3

4. NHS. Make Every Contact Count, 2014. england.nhs.uk/wpcontent/uploads/2014/06/mecc-guid-booklet.pdf (accessed 16 March 2017)

5. Fisher A, Williams K, Beeken R, Wardle J. Recall of physical activity advice was associated with higher levels of physical activity in colorectal cancer patients. BMJ Open 2015; 5(4).

6. Michie S, van Stralen MM, West R. The behaviour change wheel: A new method for characterising and designing behaviour change interventions. Implementation Science. BioMed Central; 2011 Apr 23;6(1):42.

7. Webb J, Foster J, Poulter E. Increasing the frequency of physical activity very brief advice for cancer patients. Development of an intervention using the behaviour change wheel. Public Health. Elsevier 2016;133:45-56.

8. Van Nieuwenborg L, Goossens M, De Lepeleire J, Schoenmakers B. Continuing medical education for general practitioners: a practice format. Postgraduate Medical Journal 2016; 92(1086):217-22.

9. Glasgow RE, Vogt TM, Boles SM. Evaluating the public health impact of health promotion interventions: the RE-AIM framework. Am J Public Health. 1999; $1 ; 89(9): 1322-7$.

10. Nursing Time. Macmillan Resources Hub.

https://www.nursingtimes.net/macmillan (accessed 16 March 2017) 